Quim. Nova, Vol. 33, No. 3, 566-570, 2010

\title{
EVALUACIÓN DE LA TOXICIDAD DE COBRE EN SUELOS A TRAVÉS DE BIOMARCADORES DE ESTRÉS OXIDATIVO EN Eisenia foetida
}

\author{
Hernán Gaete* \\ Departamento de Biología y Ciencias Ambientales, Facultad de Ciencias, Centro de Investigación y Gestión de Recursos Naturales, \\ Universidad de Valparaíso, Av. Gran Bretaña 1111, Valparaíso - Chile \\ María Eliana Hidalgo \\ Departamento de Bioquímica y Química, Facultad de Ciencias, Universidad de Valparaíso, Av. Gran Bretaña 1111, Valparaíso - Chile \\ Alexander Neaman y Gonzalo Ávila \\ Facultad de Agronomía, Pontificia Universidad Católica de Valparaíso, Casilla 4-D, Quillota, Chile
}

Recebido em 5/3/09; aceito em 19/8/09; publicado na web em 11/1/10

\begin{abstract}
ASSESSMENT OF COPPER TOXICITY IN SOILS USING BIOMARKERS OF OXIDATIVE STRESS IN Eisenia foetida. Copper toxicity in soil was evaluated using biomarkers of oxidative stress (catalase enzyme activity, superoxide dismutase and lipid peroxidation) in the earthworm Eisenia foetida. Agricultural topsoils from mining areas of the Aconcagua river basin were collected. Total copper concentrations were in the range of $94-959 \mathrm{mg} \mathrm{kg}^{-1}$, while the exchangeable copper concentrations were in the range

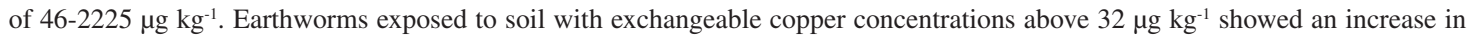
catalase activity. Parameters of antioxidant activity were more sensitive than the weight change and thus can be used as appropriate biomarkers in Eisenia foetida.
\end{abstract}

Keywords: catalase; superoxide dismutase.

\section{INTRODUCCIÓN}

Las lombrices de tierra, junto con otros organismos macrodescomponedores, están entre los organismos más importantes del suelo. Esto se debe a la capacidad de descomponer la materia orgánica, reciclamiento de nutrientes y formación de suelo. ${ }^{1,2}$ Esta importante función puede ser afectada por la presencia de elementos tóxicos en el suelo. ${ }^{3}$ Estas se caracterizan por tener un ciclo de vida en condiciones ambientales naturales de 4 años aproximadamente y en cautiverio pueden llegar como el caso de Eisenia foetida a los 15 años. ${ }^{4}$

El cobre es un importante contaminante ambiental asociado, en el caso de Chile, principalmente a las actividades mineras. ${ }^{5}$ Éste altera las características naturales de los suelos en sectores cercanos a dichas actividades, pudiendo provocar efectos adversos sobre los organismos vivos del suelo. El cobre es un micronutriente esencial para el crecimiento de los organismos, pero es requerido sólo en cantidades traza, ya que a altas concentraciones es tóxico para las células al alterar las funciones de las proteínas y consecuentemente la actividad enzimática. ${ }^{67}$ Este metal se encuentra normalmente como complejos en las proteínas de las células y, si se encuentra como ión libre, puede generar estrés oxidativo y daños en macromoléculas. ${ }^{8}$

$\mathrm{El} \mathrm{O}_{2}^{-\bullet} \mathrm{y} \mathrm{H}_{2} \mathrm{O}_{2}$ son relativamente poco reactivos, sin embargo ambos pueden interaccionar ,a través de la reacción de Fenton, con iones de metales de transición como el hierro y el cobre para producir el extremadamente reactivo $\mathrm{OH}^{*}$ considerado el más dañino de los radicales oxigenados. ${ }^{9,10}$

$$
\begin{aligned}
& \mathrm{H}_{2} \mathrm{O}_{2}+\mathrm{Fe}^{++} \rightarrow \mathrm{OH}^{\bullet}+\mathrm{OH}^{-}+\mathrm{Fe}^{+++} \\
& \mathrm{H}_{2} \mathrm{O}_{2}+\mathrm{O}_{2}^{-\cdot} \rightarrow \mathrm{OH}^{\cdot}+\mathrm{OH}^{-}+\mathrm{O}_{2}
\end{aligned}
$$

reacción de Fenton

reacción de Haber - Weiss

Los metales pesados constituyen una fuente importante de estrés oxidativo, aumentan la actividad antioxidante de las enzimas SOD,

\footnotetext{
*e-mail: hernan.gaete@uv.cl
}

catalasa y peroxidasas en plantas. ${ }^{11}$ Por ejemplo, la toxicidad del cadmio causa estrés oxidativo produciendo cambios en las enzimas antioxidantes, ${ }^{12}$ aumenta la lipoperoxidación, reflejándose en un aumento en la concentración de malondialdehído. ${ }^{13,14}$ Sin embargo, se han observado diferentes efectos en la actividad de enzimas antioxidantes, incluyendo SOD, peroxidasa (POD), catalasa (CAT) y ascorbato peroxidasa (APX), atrapando especies reactivas de oxígeno. ${ }^{13}$ Debido a sus propiedades redox, el cobre puede catalizar la formación de radicales libres muy dañinos tales como especies reactivas de oxígeno y peróxidos, causando desbalance oxidativo..$^{15-17}$

La generación de radicales libres provoca la respuesta del sistema defensivo antioxidante..$^{18,19} \mathrm{Un}$ efecto dañino de las especies reactivas de oxígeno es la peroxidación lipídica de la membrana. La lipoperoxidación está relacionada con el aumento de la permeabilidad de iones, uniones entre lípidos y polipéptidos y la inactivación de proteínas y enzimas de la membrana. ${ }^{19} \mathrm{El}$ estrés oxidativo es básicamente la consecuencia de un desequilibrio entre producción de radicales libres y la capacidad antioxidante de un organismo. ${ }^{20}$

El uso de índices de estrés subletal como biomarcadores es un enfoque alternativo y potencialmente muy útil para evaluar la respuesta de un organismo o población a los contaminantes. La inducción de ciertas enzimas en reacciones de biotransformación y detoxificación de contaminantes ha sido demostrada en diferentes organismos (invertebrados y plantas tanto acuáticas como terrestres) y su aplicación como herramienta para evaluar la calidad de aguas y suelos ha sido propuesta recientemente..$^{21,22}$

Los suelos agrícolas de la cuenca del río Aconcagua (Región de Valparaíso, Chile) presentan elevadas concentraciones de cobre en comparación con áreas sin actividad minera. ${ }^{23,24}$ Los organismos expuestos a estos suelos podrían tolerar los efectos del cobre a través de mecanismos de protección antioxidante. En esta investigación se propuso evaluar la toxicidad de cobre en suelos agrícolas cercanos a fuentes de contaminación minera a través del uso de bioensayos de toxicidad y biomarcadores de estrés oxidativo en la lombriz Eisenia foetida. 


\section{PARTE EXPERIMENTAL}

\section{Caracterización físico-química de suelos}

Se tomaron 6 muestras de suelos agrícolas en diferentes localidades en la cuenca del río Aconcagua. Los sitios de muestreo se establecieron en áreas cercanas a actividades mineras, el control correspondió a la estación sin actividad minera. Las muestras de suelo $(10 \mathrm{~kg})$ fueron tomadas entre 0 a $20 \mathrm{~cm}$ de profundidad, removiendo la vegetación existente, fueron secados a $60{ }^{\circ} \mathrm{C}$ durante 2 días, posteriormente desagregados con un mortero de granito y finalmente tamizados a través de un tamiz de $2 \mathrm{~mm}$ de apertura. Para el análisis de cobre total, una porción de cada muestra fue molida fino en un mortero de ágata.

La materia orgánica fue determinada según Sadzawka et al. ${ }^{25} \mathrm{El}$ cobre total fue determinado por espectroscopia de absorción atómica, después de la digestión ácida de las muestras con una mezcla de ácidos fluorhídrico y perclórico. ${ }^{26}$

Para el aseguramiento y control de calidad de los resultados, se analizaron dos muestras de referencia: GBW 07312 (sedimento de río) y IAEA-SL-1 (sedimento lacustre) del International Atomic Energy Agency. Los valores de referencia fueron $1230 \pm 12$ y $30 \pm$ $5,0 \mathrm{mg} \mathrm{kg}^{-1}$ mientras que los obtenidos fueron $1250 \pm 14$ y $28,8 \pm 0,7$ $\mathrm{mg} \mathrm{kg}^{-1}$, respectivamente. Es decir, la desviación entre los valores de referencia y los obtenidos fue menor al $5 \%$.

Las soluciones de sales neutras se consideran como las más adecuadas para simular la disponibilidad de elementos traza a las plantas y los organismos del suelo. ${ }^{26}$ En este estudio, se usó $\mathrm{KNO}_{3}$ $0,1 \mathrm{~mol} \mathrm{~L}^{-1}$ como extractor. ${ }^{25}$ En este extracto, se determinaron $\mathrm{pH}$, cobre intercambiable y $\mathrm{pCu}^{+2}\left(\mathrm{pCu}^{+2}=-\log \left[\mathrm{Cu}^{+2}\right]\right.$, donde $\left[\mathrm{Cu}^{+2}\right]$ es la actividad del ión libre $\mathrm{Cu}^{+2}$ ). Las concentraciones de cobre en solución fueron determinadas por espectroscopia de absorción atómica. El cobre libre $\left(\mathrm{Cu}^{2+}\right)$ se determinó usando un electrodo de ión selectivo. ${ }^{27}$ La determinación de los parámetros se realizó al inicio y al final de los periodos de exposición de los organismos en los suelos.

\section{Biomarcadores en tejidos de $E$. foetida medidos a los 15 y 45 días de exposición}

\section{Superoxido dismutasa (SOD)}

Se determinó espectrofotométricamente la reducción del citocromo $\mathrm{C}$ en presencia del sistema Xantina-oxidasa con un equipo CECIL, CE 2041, 2000 Series. La cinética de formación de citocromo reducido se evaluó a partir de los datos de absorbancia a $550 \mathrm{~nm}$. Se calculó la constante de velocidad. La actividad de SOD se obtuvo por interpolación en una curva de calibración de enzima pura. ${ }^{28}$

\section{Determinación de proteínas}

Se basó en la determinación espectrofotométrica de un compuesto coloreado estable, hidrazona, resultante de la reacción de los grupos carbonilos de las proteínas oxidadas y el reactivo 2,4-dinitrofenilhidrazina (DNPH). La absorbancia de las muestras tratadas con DNPH se determinó a $372 \mathrm{~nm}$. El contenido de carbonilos se calculó en base al coeficiente de extinción molar de las hidrazonas $\left(21000 \mathrm{M}^{-1} \mathrm{~cm}^{-1}\right)$ y expresado en nmoles $\mathrm{mg}^{-1}$ de proteínas. ${ }^{29}$

\section{Determinación de productos reactivos al ácido tiobarbitúrico (TBARS)}

La lipoperoxidación produce aldehídos siendo el más abundante el malondialdehído (MDA). Estos productos reaccionan con el ácido tiobarbitúrico generando compuestos coloreados que pueden ser cuantificados por espectrofotometría óptica en la región visible del espectro de absorción a $535 \mathrm{~nm}$. Una vez separadas las proteínas de la muestra, por precipitación con ácido tricloroacético (30\% p/v), el sobrenadante se hizo reaccionar con ácido tiobarbitúrico $(0,67 \%)$, calentando en baño maría hirviente. Se determinó la intensidad del pico a $535 \mathrm{~nm}$. Los datos se expresan como nmoles $\mathrm{mg}^{-1}$ de MDA calculados mediante una curva de calibración obtenida con MDA puro. ${ }^{30}$

\section{Actividad catalasa}

La determinación de la actividad enzimática de catalasa fue realizada a través de la cinética de degradación de peróxido de hidrógeno, la que es acelerada en presencia de la enzima, según la siguiente reacción:

$2 \mathrm{H}_{2} \mathrm{O}_{2} \underset{\mathrm{CAT}}{\longrightarrow} 2 \mathrm{H}_{2} \mathrm{O}+\mathrm{O}_{2}$

La cinética se sigue espectrofotométricamente por lecturas de absorbancia a $240 \mathrm{~nm}$. Con los datos de absorbancia, se determina la constante de velocidad y a partir de ésta se obtiene la actividad de catalasa, interpolando en una curva de calibración de enzima pura (catalasa de eritrocitos humanos) y procediendo luego a la normalización de acuerdo a la cantidad de hemoglobina de cada muestra. ${ }^{31}$

\section{Bioensayos de toxicidad}

Para determinar la toxicidad crónica, se llevaron a cabo bioensayos con E. foetida obtenida del centro de cultivo de la Facultad de Agronomía de la Pontificia Universidad Católica de Valparaíso, según métodos estandarizados por la Organization for Economic Cooperation and Development. ${ }^{32}$ Para ello, en contenedores de vidrio de $750 \mathrm{~mL}$ (unidad experimental) se colocó $500 \mathrm{~g}$ de suelo ajustando la humedad a $40 \%$ p/p en cada muestra. En el bioensayo, se incubaron diez lombrices adultas de E. foetida (con clitelium visible), anteriormente lavadas con agua destilada, secadas en papel filtro y pesadas individualmente. Se consideraron 8 réplicas. Como alimento se aplicó $5 \mathrm{~g}$ de estiércol de vaca humedecido con $5 \mathrm{~mL}$ de agua destilada. Para acostumbrar las lombrices al medio, se las alimentó con dicho alimento una semana antes de la realización del bioensayo. Al término de las 4 semanas de exposición, se determinaron los pesos promedios. Los bioensayos se realizaron bajo condiciones controladas, la temperatura ambiental oscilo entre de $22-24{ }^{\circ} \mathrm{C}$, con una iluminación de 200 lux y un fotoperíodo de $12 \mathrm{~h}$ de luz y $12 \mathrm{~h}$ de oscuridad. La humedad fue mantenida por medio de la aplicación de $40 \mathrm{~mL}$ de agua destilada una vez a la semana.

\section{Bioacumulación}

La determinación de las concentraciones de cobre en el tejido de las lombrices se realizó de acuerdo a Ma. ${ }^{33}$ Veinte individuos por cada tratamiento fueron elegidos aleatoriamente, fueron lavados y dejados por $48 \mathrm{~h}$ en placas de Petri con papel de filtro, con el fin de limpiar el intestino. El papel se cambió cada día para evitar coprofagía. Luego, las lombrices se dejaron secando por 48 horas a una temperatura de $80^{\circ} \mathrm{C}$. La digestión de lombrices se realizó con una mezcla de ácidos nítrico y perclórico y clorhídrico para luego determinar el cobre por espectroscopia de absorción atómica.

\section{Análisis estadístico}

La interpretación de los resultados de los parámetros biológicos obtenidos con los suelos de las diferentes localidades, fue realizada mediante el análisis de varianza y de correlaciones con el programa Systat 5.0. En el caso que el análisis de varianza (prueba F) detectó diferencias significativas entre los tratamientos, se realizó el análisis de comparaciones múltiples de la prueba de Tukey a 5\% de significación y evaluaciones por medio de correlaciones de Pearson entre las respuestas biológicas y diferentes formas de cobre. 
Tabla 1. Características físico-químicas de los suelos estudiados. Se presentan concentraciones de cobre intercambiable $\left(\mathrm{en} \mathrm{KNO}_{3} 0,1 \mathrm{~mol} \mathrm{~L}^{-1}\right)$ al inicio y al término de la exposición del bioensayo. M.O. = materia orgánica

\begin{tabular}{|c|c|c|c|c|c|c|c|c|c|}
\hline \multirow{2}{*}{ Suelos } & \multicolumn{2}{|c|}{ Coordenadas } & \multirow{2}{*}{$\mathrm{MO} \%$} & \multirow{2}{*}{$\mathrm{pH}$} & \multirow{2}{*}{$\begin{array}{l}\mathrm{Cu} \text { total } \\
\mathrm{mg} \mathrm{kg}^{-1}\end{array}$} & \multirow{2}{*}{$\begin{array}{c}\mathrm{Cu} \text { intercambiable } \\
\mu \mathrm{g} \mathrm{kg}^{-1} \text { inicio }\end{array}$} & \multirow{2}{*}{$\begin{array}{c}\mathrm{Cu} \text { intercambiable } \\
\mu \mathrm{g} \mathrm{kg}^{-1} \text { termino }\end{array}$} & \multirow{2}{*}{$\begin{array}{l}\mathrm{pCu}^{2+} \\
\text { inicio }\end{array}$} & \multirow{2}{*}{$\begin{array}{l}\mathrm{pCu}^{2+} \\
\text { terminc }\end{array}$} \\
\hline & Oeste & Sur & & & & & & & \\
\hline I & $71^{\circ} 12^{\prime} 3^{\prime \prime}$ & $32^{\circ} 53^{\prime} 40^{\prime \prime}$ & 1,2 & 6,4 & 94 & bld & bld & 9,6 & 9,0 \\
\hline II & $70^{\circ} 57^{\prime} 3 \prime$ & $32^{\circ} 48^{\prime} 22^{\prime \prime}$ & 5,2 & 6,5 & 426 & bld & 32 & 9,0 & 8,1 \\
\hline III & $70^{\circ} 58^{\prime} 8^{\prime \prime}$ & $32^{\circ} 48^{\prime} 17^{\prime \prime}$ & 2,8 & 7,3 & 598 & 268 & 78 & 9,6 & 10,0 \\
\hline IV & $70^{\circ} 56^{\prime} 23^{\prime \prime}$ & $32^{\circ} 47^{\prime} 49^{\prime \prime}$ & 2,0 & 6,9 & 355 & 506 & 268 & 10,0 & 9,5 \\
\hline V & $70^{\circ} 57^{\prime} 58^{\prime \prime}$ & $32^{\circ} 48^{\prime} 29^{\prime \prime}$ & 5,6 & 7,1 & 707 & 1062 & 515 & 10,0 & 9,3 \\
\hline VI & $70^{\circ} 59^{\prime} 43^{\prime \prime}$ & $32^{\circ} 46^{\prime} 5^{\prime \prime}$ & 5,2 & 6,8 & 959 & 2225 & 669 & 7,8 & 8,3 \\
\hline
\end{tabular}

Bld: Bajo el límite de detección $\left(<30 \mu \mathrm{g} \mathrm{kg}{ }^{-1}\right)$

\section{RESULTADOS Y DISCUSIÓN}

La concentración de cobre total varió de $94 \mathrm{mg} \mathrm{kg}^{-1}$ en el suelo I hasta $959 \mathrm{mg} \mathrm{kg}^{-1}$ en el suelo VI, en tanto que, el cobre intercambiable estuvo abajo del límite de detección del método analítico EAA, en el suelo I, siendo el suelo VI el que tuvo la mayor concentración con $2225 \mu \mathrm{g} \mathrm{kg}^{-1}$ (Tabla1). El cobre intercambiable en general es más del $0,05 \%$ y puede alcanzar el $0,23 \%$ en la estación VI del cobre total. Similar relación entre cobre total e intercambiable fue encontrada al estudiar la comunidad de lombrices y la biodisponibilidad de cobre y cinc a lo largo de un gradiente de contaminación en algunas localidades en Finlandia (cobre total 53-5100 mg kg-1 y cobre intercambiable $\left.8-1200 \mathrm{mg} \mathrm{kg}^{-1}\right){ }^{34}$ En ese estudio a diferencia del presente trabajo se utilizó como agente extractor ácido acético, lo que puede explicar la diferencia en el cobre intercambiables que es más alto que en el nuestro, además de las diferencias en las características físicas y químicas de los suelos (materia orgánica, arcillas, $\mathrm{pH}$ ). Al comparar las concentraciones de cobre intercambiable al inicio y término en los experimentos se observó una disminución, lo que podría ser debido a la complejación de cobre por el estiércol de vaca agregado a los suelos, lo que podría afectar la biodisponibilidad del cobre en este tipo de pruebas estandarizadas. La materia orgánica del suelo fluctuó entre 1,2\% en la estación I y 5,6\% en la estación V. El pH de los suelos cercanos a la neutralidad (Tabla 1).

Las membranas fosfolipídicas de los organismos aeróbicos están continuamente sujetas a agresión oxidativa proveniente tanto de fuentes internas como externas; entre las externas se destaca el cobre. ${ }^{21}$ El daño lipoperoxidativo en las lombrices fue mayor a los 45 días que a los 15 días de exposición a los suelos (Figura 1). En ambos tiempos de exposición, se observó una variación significativa entre los suelos estudiados. A los 15 días de exposición, la mayor concentración de MDA fue en los suelos IV y V ( 5,2 y 5,1 nmoles $\mathrm{mL}^{-1}$ de MDA respectivamente), mientras que a los 45 días fue en los suelos III y IV (4,9 y 5,2 nmoles $\mathrm{mL}^{-1}$ de MDA, respectivamente) (Figura 1). Mayor concentraciones de cobre total e intercambiable aumentaron significativamente el daño lipídico, lo que es concordante con estudios en otras especies tales como carpas, ratas y salmón. ${ }^{21}$ Se encontró una correlación inversa entre $\mathrm{pCu}^{2+}$ y $\mathrm{MDA}\left(\mathrm{r}^{2} 0,75, \mathrm{P}<\right.$ $0.05)$ es decir, en los suelos con menores actividades de $\mathrm{Cu}^{2+}$, hubo menor daño a lípidos. Esto sugiere que esta medida del cobre sería un buen predictor del efecto tóxico del cobre.

La actividad de catalasa aumentó con el tiempo en las lombrices expuestas a suelos II, III y IV (0,22 y 0,40 U) siendo significativa en relación al suelo control I, tanto a los 15 días como a los 45 días (Figura 2). Este aumento sugiere la activación de mecanismos de protección asociados al cobre. Similar respuesta se obtuvo en Tubifex tubifex expuestos a crecientes concentraciones de cobre $(50,100 \mathrm{y}$ $200 \mu \mathrm{g} \mathrm{L}^{-1}$ ) por 7 y 15 días. ${ }^{35}$ La disminución de la actividad catalasa

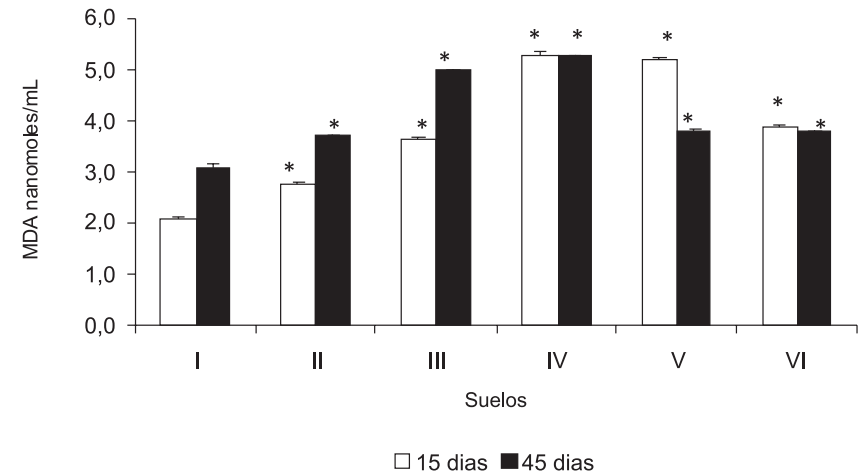

Figura 1. Daño lipoperoxidativo en E. foetida expuestas a suelos agrícolas provenientes de áreas con actividad minera durante 15 y 45 días, valores promedio y desviación estandar $(n=4)$. *: Diferencia significativa con $s u$ respectiva estación control (estación I), $P<0,05$

en las lombrices en el suelo V y VI con la mayor concentración de cobre total e intercambiable (Tabla 1) podría explicarse probablemente por daño en esta enzima por el radical hidroxilo generado por el cobre en concentraciones muy altas. $\mathrm{El}_{2}^{-\bullet}$ a través, de la reacción de Fenton, con iones de metales de transición como el hierro y el cobre producen el radical $\mathrm{OH}^{*}$ que es el extremadamente reactivo. Los radicales hidroxilos pueden dañar las enzimas, cuando se producen por descomposición catalizada por metales unidos a proteínas de membrana. El radical hidroxilo reacciona a altas velocidades con casi todo tipo de molécula en células vivientes incluyendo los azúcares,

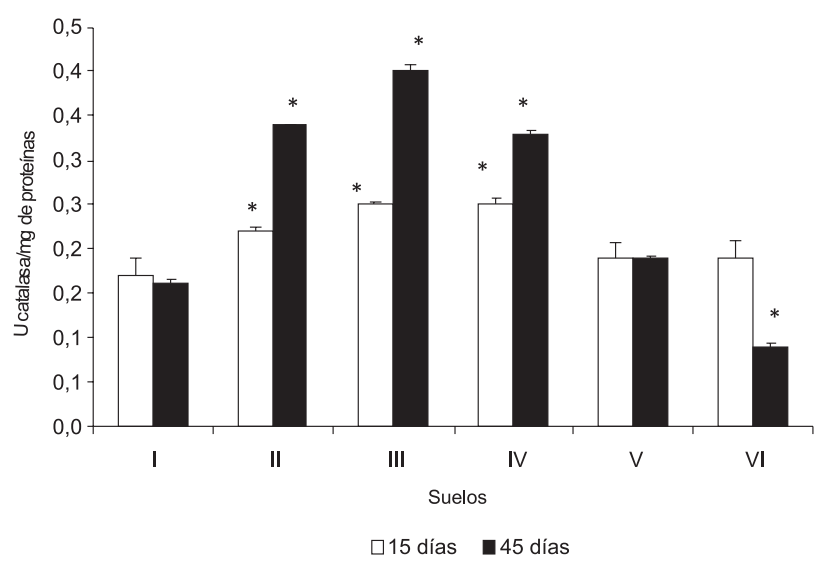

Figura 2. Comparación de la actividad enzimática de catalasa en E. foetida a los 15 y 45 días de exposición a suelos agrícolas provenientes de áreas con actividad minera, valores promedio y desviación estandar $(n=4)$. *: Diferencia significativa con su respectiva estación control (estación I), $P<0,05$ 
aminoácidos, fosfolípidos y bases del ácido desoxirribonucleico (DNA) ${ }^{9}{ }^{10}$ Lo anterior podría explicar la correlación negativa entre la actividad catalasa con $\mathrm{pCu}^{2+}\left(\mathrm{r}^{2}: 0,68, \mathrm{P}<0,05\right)$ y entre actividad catalasa y cobre intercambiable $\left(\mathrm{r}^{2}: 0,46, \mathrm{P}>0,05\right)$ aunque esta última no fue significativa. Por otra parte, este comportamiento en la enzima podría estar relacionado con la hormesis, es decir, menores concentraciones de un agente tóxico estimula la respuesta defensiva, mientras que en altas concentraciones la inhiben. ${ }^{36}$

Al comparar la actividad de superóxido dismutasa (SOD) entre los períodos de exposición (Figura 3) se observa que fue mayor a los 45 días en todos los suelos. A los 15 días de exposición no se observó diferencias significativas entre los suelos, en tanto que a los 45 días hubo diferencias significativas entre los suelos (Figura 3). Similar a lo ocurrido con la actividad de catalasa, la actividad de SOD fue mayor en las lombrices de los suelos con mayor concentración de cobre. Sin embargo, contrariamente a lo sucedido con la catalasa, la superóxido dismutasa se mantuvo más alta en el suelo VI comparada con el suelo I (Figura 3), lo cual sugiere que el mecanismo antioxidante se mantiene a esas concentraciones de cobre. El comportamiento distinto de ambas enzimas ha sido comprobado en otros estudios, denominado compensatorio. Este comportamiento se encontró al estudiar estas enzimas en larvas y adultos de Caenorhabditis elegans. ${ }^{37}$

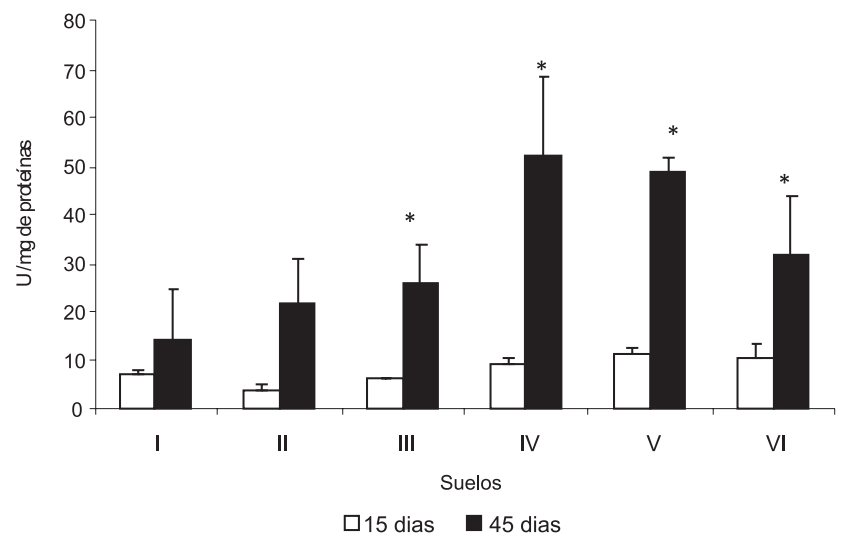

Figura 3. Comparación de la actividad enzimática superóxido dismutasa en E. foetida a los 15 y 45 días de exposición a suelos agrícolas provenientes de áreas con actividad minera, valores promedio y desviación estandar $(n=4)$. *: Diferencia significativa con su respectiva estación control (estación I), $P<0,05$

Los cambios en la biomasa de los organismos es uno de las características ecológicas sensibles. ${ }^{37}$ En este estudio, la pérdida de peso se observó en todos los suelos, excepto en el suelo III en la cual hubo un leve incremento (Figura 4). La mayor pérdida de peso se observó en el suelo V, en tanto que la menor en el suelo IV. Sin embargo, estas pérdidas de peso no fueron significativas comparadas con el suelo I (control), cuya pérdida fue $8 \%$. Esas leves diferencias podrían ser la diferencias en las características físico químicas entre los suelo. Esto sugiere que esta variable respuesta no es muy sensible a los cambios en las concentraciones de cobre en suelo. La baja correlación con las concentraciones de cobre se podría deber a la influencia del $\mathrm{pH}$, materia orgánica y textura del suelo podrían afectar la biodisponibilidad del cobre. ${ }^{38}$

Al comparar la bioacumulación entre los suelos, sólo se observa un leve incremento en el suelo VI (Figura 5). Sin embargo, no fue significativo comparado con el suelo control. Por otra parte, se observó una correlación entre cobre intercambiable y bioacumulación $\left(\mathrm{r}^{2}: 0,90, \mathrm{P}<\right.$ $0,05)$. Las concentraciones de cobre bioacumulado en las lombrices en este estudio fueron menores en comparación con las determinadas por

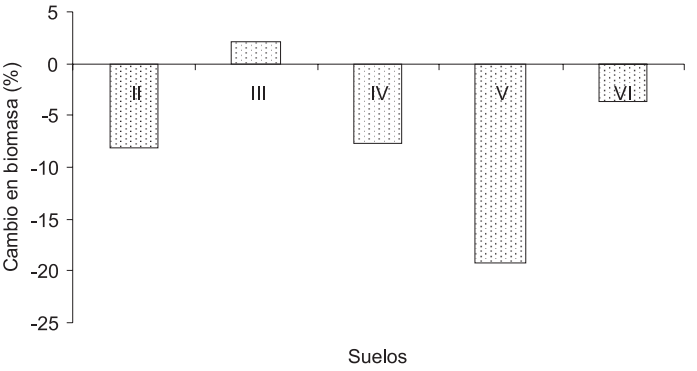

Figura 4. Cambio de peso corporal de E. foetida a las 4 semanas de exposición a suelos agrícolas provenientes de áreas con actividad minera en relación a la estación control

en Aporrectodea caliginosa, Lumbricus terrestres y Eisenia foetida, cuyas concentraciones fueron en el rango de $64-186 \mathrm{mg} \mathrm{kg}^{-1} \cdot{ }^{36}$ En ese estudio los organismos fueron colectadas en terreno, pudiendo haber estado más tiempo expuestas a cobre que en el presente estudio y tal como señala este autor la acumulación de metales en lombrices corresponde a una cinética de primer orden. Similares resultados fueron encontrados en Lumbricus rubellus colectadas en un sector contaminado con metales, cuyo rango de cobre bioacumulado fue en el rango de $8-60 \mathrm{mg} \mathrm{kg}^{-1} \cdot{ }^{33} \mathrm{Se}$ ha determinado que una concentración en tejidos de $40 \mathrm{mg} \mathrm{kg}^{-1}$ puede provocar efectos tóxicos sobre la reproducción. ${ }^{26}$ En Lumbricus terrestres se encontró concentraciones bioacumulados de cobre 8 y $74 \mathrm{mg} \mathrm{kg}^{-1}$, similar a nuestro estudio. ${ }^{39}$ En este caso, las lombrices fueron incubadas en los suelos contaminados por un período de cinco a siete semanas lo que es similar al presente estudiLa similitud en la bioacumulación del cobre en los organismos expuestos a los suelos con diferentes concentraciones de cobre en el presente estudio, podría estar relacionado con aumento de las tasas de excresión en $E$. foetida, es decir, a medida que aumenta la concentración del cobre aumenta la tasa de excresión. Al relacionar las concentraciones internas (tejido) y externas (suelos) de cobre total en la lombriz Lombricus rubellus, se encontró que ésta no fue lineal (pendiente $<1$ ), ${ }^{40}$ lo que es similar a lo encontrado en el presente estudio. En este estudio, concentración de cobre intercambiable fue mejor predictor de la bioacumulación de cobre en tejidos.

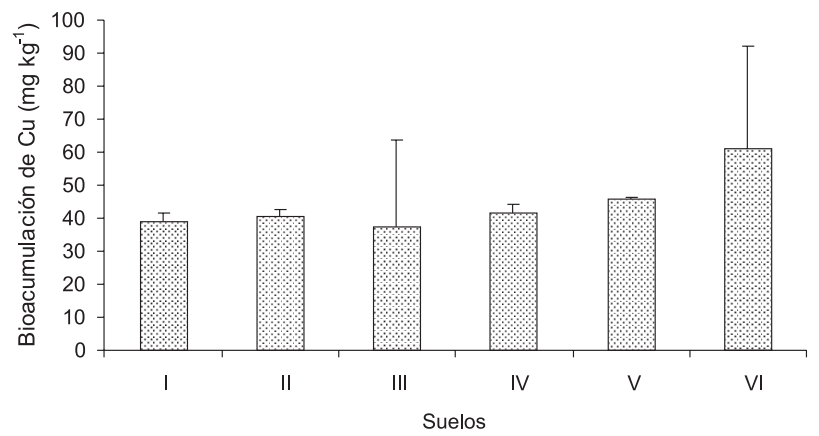

Figura 5. Bioacumulación de cobre en E. foetida al final de los bioensayos, valores promedio y desviación estándar

\section{CONCLUSIONES}

Los biomarcadores de estrés oxidativo fueron más sensibles que la pérdida de peso y bioacumulación de cobre a la variación de las concentraciones de cobre en los suelos. No hubo pérdida de peso ni bioacumulación significativa en los suelos comparados con el suelo control. Sin embargo, se observó daño lipoperoxidativo en los suelos y aumento significativo de la actividad catalasa y superóxido dismu- 
tasa en los suelos con mayor concentración de cobre. Se sugiere el uso de biomarcadores de estrés oxidativo para evaluar la toxicidad de cobre en los suelos.

\section{MATERIAL SUPLEMENTARIO}

Disponible en http://quimicanova.sbq.org.br, en arquivo.PDF, con accesso libre. Apresenta a Figura 1S. Localización de los suelos estudiados. Círculos negros muestran localización de actividades mineras.

\section{AGRADECIMIENTOS}

Esta investigación fue financiada a través del proyecto Fondecyt 1085005 y Dipuv 49/2007 de la Dirección de Investigación de la Universidad de Valparaíso.

\section{REFERENCIAS}

1. Huhta, V.; Pedobiologia 2007, 50, 489.

2. Brussaard, L.; Pulleman, M.; Oue'draogo, E.; Mando, A.; Six, J.; Pedobiologia 2007, 50, 447.

3. Spurgeon, D.; Hopking, S.; Ecotoxicol. Environ. Saf. 1996, 35, 86.

4. Giménez, R,; Della Penna, A.; Odello, E.; Agricultura técnica (Chile), 2004, 64, 347.

5. De Gregori, I.; Fuentes, E.; Rojas, M.; Pinochet, H.; Potin-Gautier, M.; J. Environ. Monit. 2003, 5, 287.

6. Adriano, D.; Trace elements in terrestrial environments: biogeochemistry, bioavailability, and risks of metals, $2^{\text {nd }}$ ed.; Springer: New York, 2001.

7. Dewes, D.; Geoffroy, L.; Vernet, G.; Popovic, R.; Aquat. Toxicol. 2005, $74,150$.

8. Ratkevicius, N.; Correa, J.; Moenne, A.; Plant, Cell and Envinonment 2003, 26, 1599.

9. Greenwald, R.; Drugs Today 1990, 26, 299.

10. Halliwell, B.; Gutteridge, J.; Free radicals in biology and medicine, $2^{\text {nd }}$ ed.; Clarendon Press: Oxford, 1989.

11. Li, M.; Hu, C. W.; Zhu, Q.; Chen, L.; Kong, Z. M.; Liu, Z. L.; Chemosphere 2006, 62, 565.

12. Sanita, L.; Toppi, di.; Gabbrielli, R.; Environ. Exp. Bot. 1999, 41, 105.

13. Chaoui, A.; Mazhoudi, S.; Ghorbal, M. H.; Ferjani, E. E. L.; Plant Sci. 1997, 127, 139.

14. Sanita, L.; Toppi, di.; Lambardi, M.; Pazzagli, L.; Cap-pugi, G.; Durante, M.; Gabbrielli, R.; Plant Sci. 1998, 137, 119.

15. Baccouch, S.; Chaoui, A.; Ferjani, E. E.; Plant Physiol. Biochem. 1998, 36,689 .
16. Richards K. D.; Schott, E. J.; Sharma, Y. K.; Davis, K. R.; Gardner, R. C.; Plant Physiol. 1998, 116, 409.

17. Weckx, J. E. J.; Clijsters, H. M. M.; Physiol. Plant 1996, 96, 506.

18. Laszczyca, P.; Relationships among indices of antioxidative activity in animals treated with selected prooxidants and antioxidants, Wydawnictwo Uniwersytetu Oellowceskiego: Katowice, 1999.

19. Vavilin, D.; Ducret, J.; Matorin, D.; Venediktov, P.; Rubin, A.; J. Photochem. Photobiol., B 1998, 42, 233.

20. Kohen, P.; Nyska, A.; Toxicol. Appl. Pharmacol. 2002, 22, 14.

21. Valavanidis, A.; Vlahogianni, T.; Dassenakis, M.; Scoullos, M.; Ecotoxicol. Environ. Saf. 2006, 64, 178.

22. Bozo, L.; Fernández, M.; López, M.; Reyes, R.; Suárez, P.; Interciencia 2007, 32, 8 .

23. Ávila, G.; Gaete, H.; Morales, M.; Neaman, A.; Pesquisa Agropecuária Brasileira 2007, 42, 435.

24. Ávila, G.; Gaete, H.; Sauve, S.; Neaman, A.; Chilean J. Agric. Res. 2009, 69, 252.

25. Sadzawka, A.; Carrasco, M.; Grez, R.; Mora, M.; Flores, H.; Neaman, A.; Métodos de análisis de suelos recomendados para los suelos de Chile, Instituto de Investigaciones Agropecuarias: Santiago, 2006.

26. Maxwell, J.; Rock and mineral analysis, Pergamon Press: Oxford, 1968.

27. Rachou, J.; Gagnon, C.; Sauvé, S.; Environ. Chem. 2000, 74, 90.

28. Chambers, D.; Parks, D.; Patterson, G.; Roy, R.; McCord, M.; Yoshida, S.; Parmley, L.; Downey, J.; J. Mol. Cell. Cardiol. 1985, 17, 145.

29. Palamada, J.; Kehrer, J.; J. Arch. Biochem. Biophys. 1992, 293, 103.

30. Aitken, R.; West, K.; J. Andrology 1990, 3, 433.

31. Aebi, H.; Methods Enzymol. 1984, 105, 121.

32. Organisation for Economic Cooperation and Development 2000; Draft guideline for the testing of chemicals: earthworm reproduction test (Eisenia foetida/andrei), Paris, 2000.

33. Ma, W.; Soil Biol. Biochem. 2005, 37, 561

34. Lukkari, T.; Taavitsainen, M.; Väisänen, A.; Haimi, J.; Ecotoxicol. Environ. Saf. 2004, 59, 340.

35. Mosleh, Y.; Paris-Palacios, S.; Biagianti-Risbourg, S.; Chemosphere 2006, 64, 121.

36. Laszczyca, P.; Augustyniak, M.; Babczyn’ska, A.; Bednarska, K.; Kafel, A.; Migula, P.; Wilczek, G.; Witas, I.; Environ. Int. 2004, 30, 901.

37. Larsen, P.; Proc. Natl. Acad. Sci. U. S. A. 1993, 90, 8905.

38. Melov, S.; Ravenscroft, J.; Malik, S.; Gill, M.; Walker, D.; Clayton, P.; Wallace, D.; Malfroy, B.; Doctrow, S.; Lithgow, G.; Science 2000, 289, 5484.

39. Kennette, D.; Hendershot, W.; Tomlin, A.; Sauvé, S.; Applied Soil Ecology 2002, 19, 191.

40. Veltman, K.; Huijbregts, M.; Vijver, M.; Peijnenburg, W.; Hobbelen, P.; Koolhaas, J.; van Gestel, C.; van Vliet, P.; Hendriks, A.; Environ. Pollut. 2007, 146, 428. 


\section{EVALUACIÓN DE LA TOXICIDAD DE COBRE EN SUELOS A TRAVÉS DE BIOMARCADORES DE ESTRÉS}

\section{OXIDATIVO EN Eisenia foetida}

\section{Hernán Gaete*}

Departamento de Biología y Ciencias Ambientales, Facultad de Ciencias, Centro de Investigación y Gestión de Recursos Naturales, Universidad de Valparaíso, Av. Gran Bretaña 1111, Valparaíso - Chile

María Eliana Hidalgo

Departamento de Bioquímica y Química, Facultad de Ciencias, Universidad de Valparaíso, Av. Gran Bretaña 1111, Valparaíso - Chile Alexander Neaman y Gonzalo Ávila

Facultad de Agronomía, Pontificia Universidad Católica de Valparaíso, Casilla 4-D, Quillota, Chile

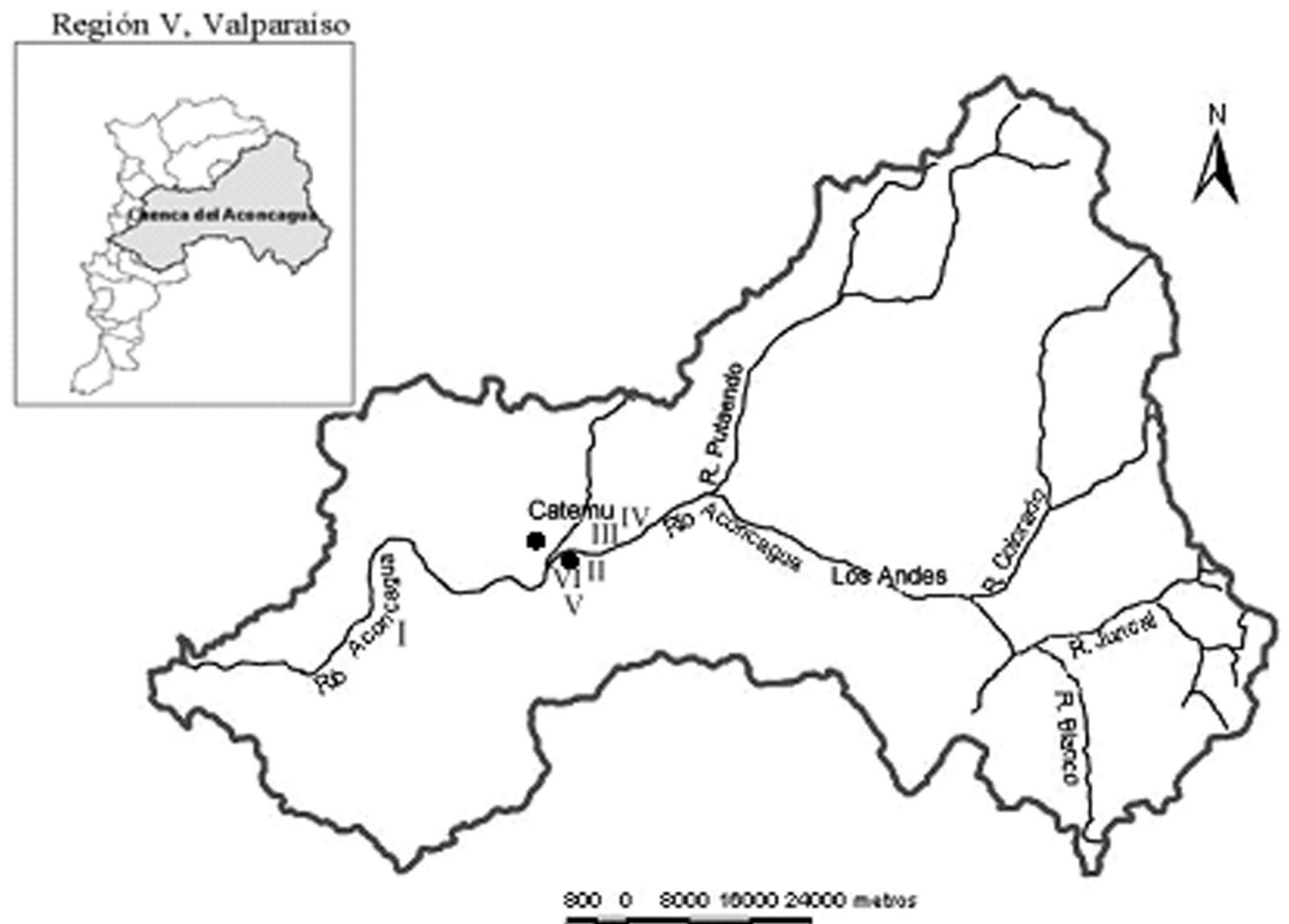

Figura 1S. Localización de los suelos estudiados. Círculos negros muestran localización de actividades mineras 